\title{
Revised 2004 International Classification of Headache Disorders: New Headache Types
}

\author{
Jonathan P. Gladstone, David W. Dodick
}

\begin{abstract}
In 1988, the International Headache Society created a classification system that has become the standard for headache diagnosis and research. The International Classification of Headache Disorders galvanized the headache community and stimulated nosologic, epidemiologic, pathophysiologic, and genetic research. It also facilitated multinational clinical drug trials that have led to the basis of current treatment guidelines. While there have been criticisms, the classification received widespread support by headache societies around the globe. Fifteen years later, the International Headache Society released the revised and expanded International Classification of Headache Disorders second edition. The unprecedented and rapid advances in the field of headache led to the inclusion of many new primary and secondary headache disorders in the revised classification. Using illustrative cases, this review highlights 10 important new headache types that have been added to the second edition. It is important for neurologists to familiarize themselves with the diagnostic criteria for the frequently encountered primary headache disorders and to be able to access the classification (www.i-h-s.org) for the less commonly encountered or diagnostically challenging presentations of headache and facial pain.
\end{abstract}

RÉSUMÉ: Classification internationale des céphalées : considérations sur de nouveaux types de céphalée. En 1988, l'International Headache Society (IHS) a créé un système de classification maintenant utilisé comme standard pour le diagnostic de la céphalée et pour la recherche. La classification des céphalées élaborée par l'IHS a galvanisé ceux qui s'intéressent à la céphalée et a stimulé la recherche nosologique, épidémiologique, physiopathologique et génétique, en plus de faciliter la réalisation d'essais thérapeutiques multinationaux qui ont permis d'établir les assises des lignes directrices actuelles de traitement. Bien qu'elle ait été critiquée, cette classification a reçu l'aval de la communauté scientifique à travers le monde. Quinze ans plus tard, l'IHS a émis une deuxième édition révisée et enrichie de la classification internationale des céphalées. Des progrès rapides et sans précédent dans ce domaine ont permis d'inclure plusieurs entités nouvelles, que ce soit des céphalées primaires ou secondaires, dans la nouvelle classification. Cette revue souligne, au moyen de cas probants, 10 nouveaux types importants de céphalée qui ont été inclus dans la deuxième édition. Il est essentiel que les neurologues se familiarisent avec les critères diagnostiques des céphalées primaires les plus fréquentes et qu'ils aient accès à cette classification (www.i-h-s.org) en ce qui concerne les céphalées et les douleurs faciales plus rares ou qui présentent un défi diagnostique.

Can. J. Neurol. Sci. 2004; 31: 304-314

Headache, by any measure, is one of the most prevalent symptoms in the general population ${ }^{1-3}$ and represents the single most common reason for outpatient visits to neurologists. ${ }^{4-6}$ Despite this, prior to the $1960 \mathrm{~s}$, there was no internationally accepted basis for classifying headaches. ${ }^{7,8}$ In 1962, the National Institute of Neurologic Diseases and Blindness formed the AdHoc Committee of the Classification of Headache and the first formal, modern-day, classification of headache syndromes was created. ${ }^{9}$ This was a two-page document that included 15 categories based on the presumed etiology of the headache syndrome (including vascular headache of the migraine type, muscle contraction headache, headache of delusional, conversion or hypochondriacal states, etc). This was the first step towards consensus-orientated terminology in the classification and diagnosis of headaches. This classification system, however, provided no operational rules or nomenclature which led to poor diagnostic precision and heterogeneous headache populations in clinical treatment trials.

In 1988, the International Headache Society (IHS) instituted

From the Department of Neurology, Mayo Clinic, Scottsdale, AZ, USA Received February 9, 2004. ACCEPTEDIN FInALFORM June 22, 2004 Reprint requests to: Jonathan P. Gladston, Department of Neurology, Mayo Clinic, 13400 E. Shea Boulevard, Scottsdale, AZ 85259, USA 
a classification system that has become the standard for headache diagnosis and research. The stated goals of the classification were to be generalizable, valid, exhaustive, reproducible, and to possess high inter- and intra-observer reliability. ${ }^{10}$ The intent was that clinicians would familiarize themselves with the common entities and be able to easily access the criteria for rarer headache types.

The system identified 12 major categories of headache, divided into two broad groups, the primary headache disorders (Categories 1-4) and the secondary headache disorders (Categories 5-12). For secondary headache disorders, the classification provides an etiological system whereby headaches are classified on the basis of an organic cause. For primary headache disorders, because the mechanisms are uncertain and objectives tests are lacking, the IHS criteria provided a descriptive system with headaches classified based on their symptom profiles.

The impact of the IHS criteria on the field of headache cannot be overstated. The criteria established a uniform terminology and consistent diagnostic criteria for a broad range of primary and secondary headache disorders and have facilitated epidemiological and genetic studies as well as multinational clinical trials that provide the basis for current research and treatment guidelines. ${ }^{8,11-15}$ While there have been criticisms, ${ }^{16-26}$ the classification received widespread international support by headache societies around the globe, was endorsed by the World Headache Organization, and has been incorporated into the International Classification of Diseases (ICD-10). ${ }^{27,28}$

Since the original edition was based largely upon personal and consensus opinion, an evidence-based revision was originally intended for publication in 1993. However, because nosologic research is tedious and time-consuming, the classification committee chose to delay revision until there was a new, meaningful body of work to act upon. ${ }^{29}$ By waiting more than a decade, the committee for the second edition was able to draw from a variety of sources including clinical descriptions, retrospective case series, patient diary studies, questionnairebased studies, longitudinal cohort studies, population and clinicbased epidemiological studies, clinical drug trials, and advances in our understanding of the neuroimaging, pathophysiology and genetics of headache disorders.

The revised version has 14 chapters (a new chapter, Headache Attributable to Psychiatric Disease, was created). There are now four chapters for primary headaches, eight for secondary headaches and two for cranial neuralgias, central and primary facial pain and other headache sub-types. The second edition identifies 45 primary and 120 secondary headache types and subtypes, and 29 causes of cranial neuralgias and central causes of facial pain. Modifications are small but significant. The second edition includes many new headache types (see Table 1) and several headache entities have been renamed (see Table 2). It is important for all neurologists to familiarize themselves with the diagnostic criteria for the commonly encountered primary headache disorders and to be able to access the classification (available online at www.i-h-s.org) in order to look up criteria, differential diagnosis and salient references for the less frequently encountered or diagnostically challenging presentations of headache and facial pain. An accurate diagnosis not only facilitates appropriate therapy, but avoids misdiagnoses which

\section{Table 1. New additions to the classification criteria}

\section{a) Primary Headaches:}

- Typical aura without headache [1.2.3]

- Cyclical vomiting [1.3.1]

- Abdominal migraine [1.3.2]

- Chronic migraine [1.5.1]

- Persistent aura without infarction [1.5.3]

- Migraine-triggered seizure [1.5.5]

- Probable chronic migraine [1.6.5]

- Episodic paroxysmal hemicrania [3.2.1]

- SUNCT[3.3]

- Hypnic headache [4.5]

- Primary thunderclap headache [4.6]

- Hemicrania continua [4.7]

- New daily persistent headache [4.8]

\section{b) Secondary Headaches:}

- Acute and chronic headache attributed to whiplash injury [5.3, 5.4]

- Postcraniotomy headache [5.7]

- Headaches attributable to dural AV fistula [6.3.3], cavernous angioma [6.3.4] or Sturge-Weber syndrome [6.3.5]

- Headaches attributable to carotid angioplasty [6.5.3], endovascular procedure [6.5.4] or angiography [6.5.5]

- Headaches attributable to other intracranial vascular disorders CADASIL[6.7.1], MELAS [6.7.2], benign angiopathy of the CNS [6.7.3], pituitary apoplexy [6.7.4]

- Headaches attributable to spontaneous low CSF pressure [7.2.3]

- Headaches attributable to epileptic seizure [7.6]

- Headaches attributable to Chiari Malformation type 1 [7.7]

- HaNDL(Syndrome of transient Headache and Neurological Deficits with cerebrospinal fluid Lymphocytosis) [7.8]

- Headaches attributable to phosphodiesterase inhibitors [8.1.2], cocaine [8.1.6], cannabis [8.1.7], histamine [8.1.8], CGRP[8.1.9]

- Headaches as an acute adverse event attributed to medications used for other indications [8.1.10]

- Headaches induced by acute substance use or exposure [8.1.11]

- Headaches as an adverse event attributed to chronic medication [8.3]

- Exogenous hormone-induced headache [8.3.1]

- Headaches attributable to HIV/AIDS [9.3]

- Chronic postinfection headache [9.4]

- Diving headache [10.1.2]

- Headaches attributable to hypothyroidism [10.4]

- Headaches attributable to fasting [10.5]

- Cardiac cephalgias [10.6]

- Headaches attributable to other disorders of homeostasis [10.7]

- Headaches attributable to craniocervical dystonia [11.2.3]

- Headaches attributable to ocular inflammatory disorders [11.3.4]

- Headaches attributable to somatisation disorder [12.1]

- Headaches attributable to psychotic disorder [12.2]

- Nasociliary neuralgia [13.5]

- Supraorbital neuralgia [13.6]

- Trigeminal branch neuralgias [13.7]

- Facial pain due to multiple sclerosis [13.18.3]

- Persistent idiopathic facial pain [13.18.4]

- Burning mouth syndrome [13.18.5]

- Headache unspecified [14.2] 


\section{Table 2. Headache disorders which have been renamed in the new classification criteria}

\section{FormerName}

- Hemiplegic migraine

- Basilar migraine

- Migrainous disorder

- Episodic tension-type headache

- Idiopathic stabbing headache

- Benign cough headache

- Benign exertional headache

- Headache associated with sexual activity

- Benign intracranial hypertension

- Headache induced by chronic substance abuse

- Idiopathic trigeminal neuralgia

\section{Current Name}

- Familial hemiplegic [1.2.4] \& sporadic hemiplegic migraine [1.2.5]

- Basilar-type migraine [1.2.6]

- Probable migraine without and with aura [1.6.1., 1.6.2]

- Infrequent [2.1] and frequent [2.2] episodic tension-type headache

- Primary stabbing headache [4.1]

- Primary cough headache [4.2]

- Primary exertional headache [4.3]

- Primary headache associated with sexual activity [4.4] (pre-orgasmic and orgasmic)

- Idiopathic intracranial hypertension (IIH) [7.1.1] and IIH secondary to metabolic cause [7.1.2]

- Medication-overuse headache [8.2]

- Classical trigeminal neuralgia [13.1.1] may lead to inappropriate and sometimes harmful medical or surgical treatment. ${ }^{30}$ Using illustrative case descriptions, we hope to familiarize neurologists with some of the newly introduced and important headache disorders that they will undoubtedly encounter in clinical practice. We also hope to encourage clinicians to utilize the criteria whenever possible in an effort to continue to refine our knowledge of both primary and secondary headache disorders.

\section{Chronic migraine [1.5.1 $]^{29}$}

Description:

Migraine headache occurring on 15 or more days per month for more than three months in the absence of medication overuse.

Diagnostic criteria:

A. Headache fulfilling criteria $C$ and D for 1.1 Migraine without aura on $\geq 15$ days/month for $>$ three months

B. Not attributed to another disorder ${ }^{1,2}$

Note:

1. History and physical and neurological examinations do not suggest any of the disorders listed in groups 5-12, or history and/or physical and/or neurological examinations do suggest such disorder but it is ruled out by appropriate investigations, or such disorder is present but headache does not occur for the first time in close temporal relation to the disorder.

2. When medication overuse is present and fulfils criterion $\mathrm{B}$ for any of the subforms of 8.2 Medication-overuse headache, it is uncertain whether this criterion $B$ is fulfilled until two months after medication has been withdrawn without improvement.

Figure 1a: Chronic migraine [1.5.1 $]^{29}$

\section{Primary Headache Disorders}

\section{Case \#1 - Chronic Daily Headache and Analgesic Overuse}

This 48-year-old woman has had migraine with aura since age 12 . Attacks initially occurred with a frequency of once per month but over the past 10 years have gradually increased in frequency. Over the past four years, she has had near daily mild-moderate itensity headache with episodes of disabling headache occurring one to two times each week. In the last few years, 'migrainous'features such as nausea and emesis have diminished, but photophobia and phonophobia are quite prominent and present to some extent almost daily. Over at least the past four years, she has been using up to four butalbital containing analgesics almost daily. She treats a more disabling headache with an oral triptan, but this will invariably only provide incomplete relief. She is otherwise healthy, neurological examination is normal, and she has had two brain MRI scans over the past five years which were normal.

This woman has a three decade history of migraine without aura [1.1] which evolved into a daily headache with migrainous features, with superimposed episodes of disabling migraine headache. She is otherwise well and there are no red flags for a secondary cause other than her daily consumption of a butalbitalcontaining analgesic for four years. As no chronic form of

\section{Probable Chronic migraine $[1.6 .5]^{29}$}

Diagnostic criteria:

A. Headache fulfilling criteria $C$ and $D$ for 1.1 Migraine without aura on $\geq 15$ days/month for $>$ three months

B. Not attributed to another disorder ${ }^{1}$ but there is, or has been within the last two months, medication overuse fulfilling criterion B for any of the subforms of 8.2 Medication-overuse headache

Figure 1b: Probable chronic migraine [1.6.5 $]^{29}$ 
Medication-overuse heAdACHE $[8.2 .7]^{29}$

Triptan-overuse headache ${ }^{29}$

Diagnostic criteria:

A. Headache present on $>15$ days/month with at least one of the following characteristics and fulfilling criteria $\mathrm{C}$ and $\mathrm{D}$ :

1. predominantly unilateral

2. pulsating quality

3. moderate or severe intensity

4. aggravated by or causing avoidance of routine physical activity (eg, walking or climbing stairs)

5. associated with at least one of the following:

a) nausea and/or vomiting

b) photophobia and phonophobia

B. Triptan intake (any formulation) on $\geq 10$ days/month on a regular basis for $\geq$ three months

C. Headache frequency has markedly increased during triptan overuse

D. Headache reverts to its previous pattern within two months after discontinuation of triptan.

Analgesic-overuse headache ${ }^{29}$

Diagnostic criteria:

A. Headache present on $>15$ days/month with at least one of the following characteristics and fulfilling criteria $\mathrm{C}$ and $\mathrm{D}$ :

1. bilateral

2. pressing/tightening (nonpulsating) quality

3. mild or moderate intensity

B. Intake of simple analgesics on $\geq 15$ days $/$ month $^{1}$ for $>3$ months

C. Headache has developed or markedly worsened during analgesic overuse

D. Headache resolves or reverts to its previous pattern within 2 months after discontinuation of analgesics

\section{Opioid-overuse headache ${ }^{29}$}

\section{Diagnostic criteria:}

A. Headache present on $>15$ days/month fulfilling criteria $C$ and $D$

B. Opioid intake on $\geq 10$ days/month for $>3$ months

C. Headache develops or markedly worsens during opioid overuse

D. Headache resolves or reverts to its previous pattern within 2 months after discontinuation of opioid

\section{Combination medication-overuse headache ${ }^{29}$}

Diagnostic criteria:

A. Headache present on $>15$ days/month with at least one of the following characteristics and fulfilling criteria $\mathrm{C}$ and $\mathrm{D}$ :

1. bilateral

2. pressing/tightening (nonpulsating) quality

3. mild or moderate intensity

B. Intake of combination medications ${ }^{1}$ on $\geq 10$ days/month for $>3$ months

C. Headache develops or markedly worsens during combination medication overuse

D. Headache resolves or reverts to its previous pattern within 2 months after discontinuation of combination medication

Note:

1. Combination medications typically implicated are those containing simple analgesics combined with opioids, butalbital and/or caffeine

Figure 1c: Medication-overuse headache [8.2.7 $]^{29}$ migraine was recognized in the previous classification scheme, a common criticism was that it was challenging to classify patients such as these with a unifying diagnosis. ${ }^{17-20}$ Mathew $^{31}$ created the term transformed migraine and Silberstein and colleagues ${ }^{19}$ created and field-tested diagnostic criteria. However, there has been considerable confusion as to how to take into account the effects of frequent and often near-daily use of analgesics. Now, utilizing the revised classification criteria, the appropriate diagnosis would be probable chronic migraine [1.6.5] and probable medication overuse headache [8.2.7] (see Figure 1a-c).

The term probable is utilized to recognize that for any given individual's chronic daily headache $(\mathrm{CDH})$, it is typically impossible to predict whether the daily headache is attributable to the biological evolution of migraine as a progressive disorder, 'rebound'from overused analgesics, or a combination of the two. Currently, epidemiological, pathophysiologic and clinical evidence supports both the occurrence of chronic migraine arising de novo without analgesic overuse as well as daily headache developing as a consequence of frequent or daily use of acetominophen, combination analgesics with caffeine or butalbital, opioids and/or triptans. ${ }^{32-36}$ The new classification provides criteria for the monthly consumption of each agent required to precipitate medication-overuse headache (see Figure 1c). Following the withdrawal from the overused medication(s), if the daily headache converts to an episodic pattern of headache ( $<15$ days per month) within two months, the primary diagnosis should be medication-overuse headache and episodic migraine. If the daily headache persists, the diagnosis is chronic migraine.

There are several features of the IHS definition for chronic migraine and medication overuse headache that have generated considerable controversy and which will be problematic in clinical practice. The first is the need to withdraw the overused analgesics as the only therapeutic maneuver to make an accurate diagnosis. In practice, this will rarely be possible as patients are invariably started on a treatment plan which includes behavioral and lifestyle modifications, a change in acute therapy, and the addition of one or more preventive medications. A positive outcome at two months can, therefore, not be attributed to the withdrawal of an overused analgesic alone. Secondly, it is unclear whether most patients encountered in clinical practice will have IHS-defined migraine on more than 15 days per month. In many instances, headache diaries are either not kept by patients or they are not of sufficient detail to make this determination during the first clinical encounter. It is also unlikely, that those patients who do have more than 15 migraine days per month, are using analgesics on less than 10 days per month. Furthermore, many patients may use multiple different acute treatments, each less than 10 days per month, but when combined, collectively, acute treatment is utilized more than 10 days per month. No criteria exist for this particular circumstance. The validity of the criteria for chronic migraine and medicationoveruse headache will, therefore, require extensive field-testing and further revisions are anticipated.

\section{Case \#2 - Tic with tears.}

This 36-year-old woman has multiple daily attacks of headache for two months. On a daily basis, she has 10-30 attacks lasting 15-120 seconds. The pain is a stabbing discomfort located exclusively in a right peri-orbital distribution. Attacks are accompanied by prominent 


\section{SHORT-LASTING UNILATERAL NEURALGIFORM HEADACHE ATTACKS} WITH CONJUNCTIVAL INJECTION AND TEARING (SUNCT) [3.3 $]^{29}$

\section{Description:}

This syndrome is characterized by short-lasting attacks of unilateral pain that are much briefer than those seen in any other TAC and very often accompanied by prominent lacrimation and redness of the ipsilateral eye.

\section{Diagnostic criteria:}

A. At least 20 attacks fulfilling criteria B-D

B. Attacks of unilateral orbital, supraorbital or temporal stabbing or pulsating pain lasting 5-240 seconds

C. Pain is accompanied by ipsilateral conjunctival injection and lacrimation

D. Attacks occur with a frequency from 3 to 200 per day

E. Not attributed to another disorder ${ }^{1}$

Figure 2: Short-lasting unilateral neuralgiform headache attacks with conjunctival injection and tearing (SUNCT) $[3.3]^{29}$

ipsilateral tearing and conjunctival injection. Neurological examination is normal.

This woman has an interesting and dramatic headache. On a daily basis she has multiple paroxysmal attacks of intense firstdivision trigeminal pain associated with cranial autonomic symptoms. This trigeminal autonomic cephalgia is referred to as SUNCT [3.3 - short-lasting, unilateral, neuralgiform headache attacks with conjunctival injection and tearing] (see Figure 2). ${ }^{37}$ This is an uncommon but disabling primary headache disorder with neuralgiform pain typically located unilaterally in the ophthalmic division of the trigeminal nerve. The reported mean age of onset is 50 years. The presence of robust autonomic features, the lack of a refractory period, and the presence of only first-division trigeminal pain, helps to differentiate SUNCTfrom trigeminal neuralgia. ${ }^{37,38}$ The short duration excludes cluster headache (typically 15-180 minutes untreated) and episodic paroxysmal hemicrania (attacks typically 2-30 minutes). It is not unreasonable, especially if diagnostic uncertainty exists, to initiate a therapeutic trial with indomethacin (to exclude an indomethacin-responsive headache such as episodic paroxysmal hemicrania). ${ }^{37,39}$ Lamotrigine, gabapentin, and topiramate are sometimes effective for patients with SUNCT although the response is often not as complete as seen with trigeminal neuralgia. However, prior to making a diagnosis of primary SUNCT, mass lesions, often located in the posterior fossa or near the sella turcica, need to be excluded with a gadoliniumenhanced brain MRI. ${ }^{37}$

\section{Case \#3 - The Continuous Side-Locked Headache}

This 36-year-old woman has had a persistent right-sided headache for three years. The headache began insidiously and in the absence of a prior history of migraine. The headache is continuous, rated $6 / 10$ in severity with periodic exacerbations reaching $9 / 10$. At the height of the pain, she notes stabbing pain in the right temple, mild ipsilateral lacrimation and clear rhinorrhea during many attacks. She has seen three family doctors, two neurologists, an ENT, allergist, a chiropractor, an acupuncturist and a homeopath. Her pain has been refractory to over-
Hemicrania continua [4.7] ${ }^{29}$

Description:

Persistent strictly unilateral headache responsive to indomethacin.

Diagnostic criteria:

A. Headache for $>3$ months fulfilling criteria $B-D$

B All of the following characteristics:

1. unilateral pain without side-shift

2. daily and continuous, without pain-free periods

3. moderate intensity, but with exacerbations of severe pain

C. At least one of the following autonomic features occurs during exacerbations and ipsilateral to the side of pain:

1. conjunctival injection and/or lacrimation

2. nasal congestion and/or rhinorrhoea

3. ptosis and/or miosis

D. Complete response to therapeutic doses of indomethacin

E. Not attributed to another disorder

Figure 3: Hemicrania continua [4.7] $]^{29}$

the-counter analgesics, triptans and prescription opioids. Neurologic examination and MRI of the brain with gadolinium is normal.

This woman presents with a continuous, unilateral headache of several years duration which has been refractory to medications. A secondary cause appears unlikely given the longstanding duration without progression, and the normal examination and neuroimaging. This patient has hemicrania continua [4.7] - a trigeminal-autonomic cephalgia which is characterized by continuous pain (no remission periods or painfree intervals between attacks) (Figure 3). ${ }^{40,41}$ Hemicrania continua is an uncommon primary headache disorder typically affecting women $(5: 1)$ in their third or fourth decade of life and is characterized by a continuous, moderate to severe, unilateral headache that is punctuated by exacerbations of severe pain and ipsilateral autonomic features (tearing, nasal congestion, conjunctival injection, ptosis etc). ${ }^{40,41}$ The autonomic features are typically not as prominent as with the other trigeminal autonomic cephalgias. Hemicrania continua almost invariably has a prompt and long-lasting response to indomethacin $(25 \mathrm{mg}$ TID to $75 \mathrm{mg}$ TID). Patients with intracranial and cervical lesions as well as lung neoplasms have been described with a clinical presentation indistinguishable from primary hemicrania continua. Therefore, a clinical index of suspicion is necessary and appropriate diagnostic investigations prudent. ${ }^{40-42}$

\section{Case \#4 - Thunderclap (subarachnoid hemorrhage-like) headache}

This previously healthy 44-year-old man was brought to the emergency department two weeks previously because of a headache which began suddenly while straining on the toilet. The headache was incapacitating and intensified within seconds. There was no previous headache history. Noncontrast CT was normal and a lumbar puncture performed 12 hours after headache onset revealed normal CSF content 


\section{Primary thunderclap headache [4.6 ${ }^{29}$}

Description:

High-intensity headache of abrupt onset mimicking that of ruptured cerebral aneurysm.

Diagnostic criteria:

A. Severe head pain fulfilling criteria $B$ and $C$

B. Both of the following characteristics:

1. sudden onset, reaching maximum intensity in $<1$ minute

2. lasting from 1 hour to 10 days

C. Does not recur regularly over subsequent weeks or months ${ }^{1}$

D. Not attributed to another disorder ${ }^{2}$

Notes:

1. Headache may recur within the first week after onset.

2. Normal CSF and normal brain imaging are required.

Figure 4: Primary thunderclap headache $[4.6]^{29}$

and pressure. Eighteen hours after headache onset, his headache improved and he was discharged. Two days later, he developed recurrence of an identical sudden incapacitating headache while sitting watching television. He returned to the emergency department and was admitted to hospital. A gadolinium-enhanced brain MRI, MRA, MRV and cerebral angiogram were normal. Once again the headache resolved after about 48 hours and has not recurred.

Thunderclap headache (TCH) is a general term referring to any severe headache of sudden onset in which the pain reaches maximum intensity within seconds or minutes. ${ }^{43,44}$ The secondary causes of TCH include subarachnoid hemorrhage, cerebral venous thrombosis, arterial dissection, hypertensive crisis / encephalopathy, pituitary apoplexy, and spontaneous intracranial hypotension. Other reports have suggested an association between $\mathrm{TCH}$ and unruptured intracranial aneurysms, ${ }^{45,46}$ although it is debated whether unruptured aneurysms can present with thunderclap headache or whether their association is coincidental, especially given the prevalence of intracranial aneurysms in the general population (3-6\%). At this time, therefore, with the high resolution of magnetic resonance and CT angiography, catheter angiography may not be necessary in patients with primary TCH with a bloodless CT, MRI, and CSF.

In patients with $\mathrm{TCH}$ whose neurological examination is normal and thorough investigations (CSF, CT, MRI, MRA, MRV) have adequately ruled out a secondary cause, a diagnosis of primary TCH [4.7] is appropriate (see Figure 4). Primary TCH represents a distinct and sometimes recurrent primary headache disorder (previously referred to as benign or idiopathic TCH, benign vascular headache, migrainous vasospasm, crash migraine, benign sexual (coital) headache type II, benign isolated cerebral vasculitis or benign angiopathy of the nervous system). Because clinical features cannot reliably distinguish primary $\mathrm{TCH}$ from symptomatic $\mathrm{TCH}$, careful diagnostic investigation is necessary for all patients with $\mathrm{TCH}$, and primary $\mathrm{TCH}$ must always be considered a diagnosis of exclusion.

The natural history of primary $\mathrm{TCH}$ appears benign. According to a retrospective study of 71 patients with TCH and negative CT and lumbar puncture, no patient had a subarachnoid

\author{
HYPNIC HEADACHE $[4.5]^{29}$ \\ Description: \\ Attacks of dull headache that always awaken the patient \\ from asleep. \\ Diagnostic criteria: \\ A. Dull headache fulfilling criteria B-D \\ B. Develops only during sleep, and awakens patient \\ C. At least two of the following characteristics: \\ 1. occurs $>15$ times per month \\ 2. lasts $\geq 15$ minutes after waking \\ 3. first occurs after age of 50 years \\ D. No autonomic symptoms and no more than one of \\ nausea, photophobia or phonophobia \\ E. Not attributed to another disorder ${ }^{1}$
}

Figure 5: Hypnic headache [4.5 $]^{29}$

hemorrhage during an average follow up of 3.3 years. ${ }^{47}$ Similarly, in four prospective studies involving 225 patients with $\mathrm{TCH}$ and negative CT and lumbar puncture, there were no cases of subarachnoid hemorrhage or sudden death during one year or more of follow-up. ${ }^{44}$ The mechanism underlying TCH in these patients is incompletely understood.

\section{Case \#5 - 'Alarm-clock'headaches}

This 62-year-old woman has been awakened from sleep four to five times per week, over the past two years, by a dull, bilateral, frontal headache. The headaches occur predictably at 2:00am. The headaches are not associated with photophobia, phonophobia, nausea, emesis, or cranial autonomic symptoms. The patient reports the need to rise from bed to get relief from the headache. Each headache lasts 30 minutes to one hour untreated and she is able to fall back to sleep afterwards, although the headache can sometimes occur again later in the morning at about 5:00am. The headache does not occur during the day, except for several attacks which occurred while the patient was taking a nap. She and her husband deny a history of significant snoring or sleep apnea. Neurological examination is normal.

Nocturnal headaches are often considered to be a potentially ominous symptom. However, there is substantial clinical, anatomical, biological, and physiologic evidence to support an inherent association between the normal physiology of sleep and the genesis of headache in biologically predisposed individuals. $^{48}$ Notably, many primary headache disorders (cluster, migraine and chronic paroxysmal hemicrania) can wake an individual from sleep. Cluster headache in particular is characterized by a striking association with the sleep-wake cycle and other circadian biorhythms, suggesting that it may represent a centrally originating chronobiologic disorder. However, in this case, the mild-to-moderate intensity, bilateral location, short duration, absence of migrainous and cranial autonomic features renders this disorder unlikely. The diagnosis in this case is hypnic headache [4.5] - a benign, recurrent, headache disorder that occurs only during diurnal and nocturnal sleep and is more common in females and the elderly (see Figure 5). ${ }^{49}$ Hypnic headaches occur with a striking consistency, waking patients at 


\section{Syndrome of transient Headache and Neurological DEFICITS WITH CEREBROSPINAL FLUID LYMPHOCYTOSIS (HANDL) [7.8 $]^{29}$ \\ Diagnostic criteria:}

A. Episodes of moderate or severe headache lasting hours before resolving fully and fulfilling criteria $C$ and $D$

B. Cerebrospinal fluid pleocytosis with lymphocytic predominance ( $>15$ cells $/ \mu \mathrm{l}$ ) and normal neuroimaging, CSF culture and other tests for aetiology

C. Episodes of headache are accompanied by or shortly follow transient neurological deficits and commence in close temporal relation to the development of CSF pleocytosis

D. Episodes of headache and neurological deficits recur over $<3$ months

Figure 6: Syndrome of transient Headache and Neurological Deficits with cerebrospinal fluid Lymphocytosis (HaNDL) [7.8] ${ }^{(29)}$

the same time nightly or near-nightly. Hypnic headaches are typically bilateral, mild-to-moderate, and short-lasting (less than two hours). Usually there is a good response to bedtime caffeine (paradoxically), lithium carbonate, or indomethacin. ${ }^{49}$

\section{Case \#6 - Recurrent headache and focal neurological deficits}

This 34-year-old man presents with a one week history of three episodes of paresthesias involving his left face, arm, and leg lasting four to eight hours. The sensory symptoms begin in his hand, and evolve gradually to involve other body parts over a five-minute period. On the day of presentation, he also experienced a mild expressive aphasia, confusion, low-grade fever, and a throbbing bilateral frontal headache. Non-contrast CT and gadolinium-enhanced MRI were normal. Cerebrospinal fluid analysis revealed a white blood cell count of 135 with $90 \%$ lymphocytes, a moderately elevated protein $(300 \mathrm{mg} / \mathrm{dl})$, normal glucose, viral, bacterial, mycobacterial and fungal cultures, and an opening and closing pressure of $220 \mathrm{mmH}_{2} \mathrm{O}$ and $170 \mathrm{mmH}_{2} \mathrm{O}$. Symptoms did not recur during a six month follow-up period.

In accordance with the new classification, the appropriate diagnosis of exclusion in this case is HaNDL's syndrome - a syndrome of transient headache and neurologic deficits with cerebrospinal fluid lymphocytosis [7.8] (see Figure 6). HaNDL is a benign, self-limited headache disorder characterized by one or more (up to 12 , mean 2) episodes of moderate to severe hemicranial or bilateral headache accompanied by neurologic deficits. ${ }^{50}$ Most common neurologic symptoms are cheiro-oral numbness plus aphasia and/or hemiparesis (mean duration five hours). Up to $25 \%$ of patients have fever. Characteristically, there is a complete resolution of recurrent symptoms within three months and symptoms are absent between attacks. A cerebrospinal fluid lymphocytic pleocytosis with negative etiologic studies and negative serologic and neuroimaging evaluation are required. The etiology of HaNDL is unknown and proposed explanations include a rare variant of migraine, an infectious meningoencephalitis, or an autoimmune phenomenon. ${ }^{50}$

\section{NeW DAILY-PERSISTENT HEADACHE (NDPH) [4.8 $]^{29}$}

Description:

Headache that is daily and unremitting from very soon after onset (within 3 days at most). The pain is typically bilateral, pressing or tightening in quality and of mild to moderate intensity. There may be photophobia, phonophobia or mild nausea.

Diagnostic criteria:

A. Headache that, within 3 days of onset, ${ }^{1}$ fulfils criteria BD

B. Headache is present daily, and is unremitting, for $>3$ months

C. At least two of the following pain characteristics:

1. bilateral location

2. pressing/tightening (nonpulsating) quality

3. mild or moderate intensity

4. not aggravated by routine physical activity such as walking or climbing

D. Both of the following:

1. no more than one of photophobia, phonophobia or mild nausea

2. neither moderate or severe nausea nor vomiting

E. Not attributed to another disorder ${ }^{2}$

Figure 7: New daily-persistent headache (NDPH) [4.8]29

\section{Case \#7 - New Daily Persistent Headache}

This 18-year-old girl has had a continuous headache for 18 months. She recalls the exact day and time of onset - the headache began subacutely reaching maximal intensity over four to six hours. There were no obvious triggers, in particular, no head or neck trauma, systemic symptoms, new medications, herbal supplements or illicit drugs, chiropractic manipulation, or especially stressful life events. The headache is bilateral, tight or throbbing, moderate in intensity and associated with mild nausea. Physical and neurological examination as well as body mass-index were normal. Serum chemistries, spinal fluid analysis, opening and closing CSF pressure, and a noncontrast CT and MRI were normal. She had been using Tylenol with codeine four to five times per day to allow her to function at school. She had minimal relief with amitriptyline, propanalol, divalproex sodium, topiramate, one-week course of corticosteroids, or subcutaneous dihydroergotamine and sumatriptan. Despite the elimination of caffeine and analgesics, a daily headache persists.

Clinical diagnosis in this case is challenging. Initially a diagnosis of migraine was entertained but she had few migrainous symptoms and was particularly refractory to conventional acute and preventive medications. She is not obese, has no risk factors for secondary intracranial hypertension, and there were no visual symptoms suggestive of intracranial hypertension. Most other secondary causes have been excluded; however, a baseline noncontrast MRI does not rule out arterial dissection, cerebral venous sinus thrombosis, and spontaneous intracranial hypotension. We would recommend a MRI with gadolinium, MRV and MRAand if each of these are normal, the appropriate diagnosis of exclusion would be new daily persistent 
headache [4.8] (NDPH - see Figure 7). Originally described by Canadian neurologist Water Vanast and colleagues in 1985, $\mathrm{NDPH}$ is a primary headache disorder that appears to have a female predominance with a peak age of onset in the second to third decade. Little is known regarding underlying pathophysiology or appropriate treatment. ${ }^{51}$ Patients with NDPH have a rather nondescript, chronic daily headache of at least several months duration that began on a particular day (or over a few days). Patients either have no history of headache or lack a history of evolution or progression of a pre-existing headache disorder. Potential triggers appear to include a preceding viral illness and stressful life events; however, for many, no identifiable trigger is uncovered. There likely is a benign form which may resolve before presenting to neurologists and a refractory form which is particularly challenging to treat and may persist for years. ${ }^{51}$

\section{SECONDARY Headache Disorders}

\section{Case \# 8 - An unusual cause of referred headache}

This 58-year-old man has a new onset headache that began four weeks ago. He is hypertensive and a smoker but is generally well with no prior history of headache. On several occasions, shortly after beginning to walk his dog, he has developed a moderate or severe leftsided headache, accompanied by mild nausea and worsened by continued exertion. The headache typically resolves spontaneously after resting for 15-45 minutes. Noncontrast brain CT was normal.

Exertional headache disorders can be divided into primary subtypes (primary exertional headache, primary cough headache and primary headache associated with sexual activity) and secondary causes (intracranial space-occupying lesion, hydrocephalus or Chiari malformation). Another important secondary cause for neurologists to be aware of is cardiac cephalgia [10.6] (see Figure 8). ${ }^{52,53}$ It is a rare but serious headache disorder, resulting from underlying cardiac ischemia. The mechanism underlying the pain is speculative and possibilities include: referred pain from cervicothoracic sympathetic, vagal and/or somatic afferents, decreased cardiac output leading to decreased venous return and corresponding increased intracranial pressure and/or headache induced by an

\section{Cardiac cephalalgia [10.6 $]^{29}$}

Diagnostic criteria:

A. Headache, which may be severe, aggravated by exertion and accompanied by nausea and fulfilling criteria $\mathrm{C}$ and $\mathrm{D}$

B. Acute myocardial ischaemia has occurred

C. Headache develops concomitantly with acute myocardial ischaemia

B. Headache resolves and does not recur after effective medical therapy for myocardial ischaemia or coronary revascularisation

Figure 8: Cardiac cephalalgia [10.6 $]^{29}$ inflammatory or vasodilatory response from chemical mediators released by cardiac ischemia. ${ }^{52}$ Case reports have described fatal myocardial infarction where headache was the only symptom. In a few cases, headaches were replicated with simultaneous electrocardiographic changes during stress-testing and eliminated after coronary revascularization. ${ }^{52,53}$ These cases can be diagnostically challenging especially when there is no known underlying coronary artery disease or concomitant chest pain. Vigilance should be maintained, especially in those at risk for cardiac disease.

\section{Case \#9 - Just Give Me A Few Minutes to Rest}

This 54-year-old farmer has had a persistent daily headache for six months. There were no obvious precipitants or prior headache history. The headache is diffuse, dull or throbbing, and associated with mild nausea. He is typically headache-free when he wakes up in the morning but the headache characteristically returns by the time he is having breakfast. He often feels better on the weekend when he is lying around the house watching sports. Initially he noted buzzing in his ears and distortion in his hearing but that has improved. His headaches have been refractory to over the counter and prescription analgesics as well as treatment with amitriptyline and a COX-2 inhibitor. He is referred with a diagnosis of chronic tension-type headache and the family physician wonders if the headaches are stress-related

This gentleman's headache is rather nondescript and refractory. Looking at his history more closely it is apparent that his headaches are improved or eliminated when supine and consistently worsen shortly upon arising. Often this relationship is not elicited by direct questioning or is incorrectly attributed to the stress of the day and the relief from "relaxing". However, it

\section{HEADACHE ATtRibuted to SPONTANEOUS (OR IDIOPATHIC) LOW CSF PRESSURE [7.2.3 $]^{29}$ \\ Diagnostic criteria:}

A. Diffuse and/or dull headache that worsens within 15 minutes after sitting or standing, with at least one of the following and fulfilling criterion $D$ :
1. neck stiffness
2. tinnitus
3. hypacusia
4. photophobia
5. nausea

B. At least one of the following:

1. evidence of low CSF pressure on MRI (eg, pachymeningeal enhancement)

2. evidence of CSF leakage on conventional myelography, CT myelography or cisternography

3. CSF opening pressure $<60 \mathrm{~mm} \mathrm{H}_{2} \mathrm{O}$ in sitting position

C. No history of dural puncture or other cause of CSF fistula

D. Headache resolves within 72 hours after epidural blood patching

Figure 9: Headache attributed to spontaneous (or idiopathic) low CSF pressure $[7.2 .3]^{29}$ 
is also a highly characteristic feature of spontaneous intracranial hypotension defined by the IHS as headache attributed to spontaneous low CSF pressure [7.2.3] (see Figure 9). ${ }^{54}$ Clinically, this headache may resemble postdural (postlumbar) puncture headache [7.2.1]. A diffuse and dull headache typically begins within 15 minutes of rising. Patients may complain of disequilibrium, muffled hearing or tinnitus due to compensatory venous dilatation and fluctuations in endolymphatic pressure. Rarely, significant neurologic impairment has been reported due to secondary subdural fluid collections and/or compression or displacement of the posterior fossa structures. In some patients, the postural relationship may be completely lost or headaches may begin many hours after arising. Unless the characteristics of the initial headache are delineated, the diagnosis may not be suspect. ${ }^{54}$

Diagnostic investigation of choice is a gadolinium enhanced MRI which typically reveals diffuse pachymeningeal enhancement and may show subdural fluid collections, a secondary Chiari malformation, dilated cerebral and epidural venous sinuses, and an enlarged, homogenously enhancing pituitary gland. For patients who have failed conservative therapy and bed-rest, an autologous epidural blood patch may be effective. If repeated blood patches fail, it may be necessary to search for the leak with a spinal MRI, cisternography or CT myelography. Occasionally surgical repair is warranted. ${ }^{54}$

\section{Case \#10 - Valsalva-induced headache}

This 34-year-old woman has had infrequent migraines since childhood but developed a new headache five months ago. Walking briskly, going to the bathroom, coughing and sneezing have all precipitated a posterior headache lasting minutes to hours. On a few occasions, while cheering on her son at his hockey game, she experienced transient but significant blurring of her vision. She is otherwise well with no past medical history.

As outlined in case 8, exertional headaches can be divided into primary subtypes (primary cough headache [4.2], primary exertional headache [4.3], and primary headache associated with sexual activity [4.4]) and secondary causes (intracranial spaceoccupying lesion, hydrocephalus or Chiari malformation). All patients with exertional or valsalva-induced headaches require an MRI for exclusion of a secondary cause; in this case, the presence of neurologic symptoms, specifically visual blurring, raises the index of suspicion even higher. This patient's MRI demonstrated an $8 \mathrm{~mm}$ tonsillar descent and a clinical and radiographic diagnosis of a Chiari malformation (Type 1) was made (see Figure 10). A Chiari malformation is characterized by the downward displacement of the cerebellar tonsils through the foramen magnum into the upper cervical canal and other radiographic features. ${ }^{55}$

While most Chiari I malformations are congenital, it may also be acquired or secondary to spntaneous intracranial hypotension. Patients usually begin to experience symptoms in their thirties. Tonsillar herniation creates overcrowding at the craniovertebral junction. Because of the variable degrees of nervous tissue displacement, the size of the foramen magnum, and the extent of the compressed structures, clinical presentation is highly variable. ${ }^{55}$ Clinical symptoms of Chiari malformations are related to direct compression syndromes (cervical cord, cerebellum, brainstem or lower cranial nerves) and/or to CSF disturbances (hydrocephalus, syringohydromyelia, endolym-
Headache attributed to Chiari malformation type I (CM1) $[7.7]^{29}$

Diagnostic criteria:

A. Headache characterised by at least one of the following and fulfilling criterion $\mathrm{D}$ :

1. precipitated by cough and/or valsalva manoeuvre

2. protracted (hours to days) occipital and/or suboccipital headache

3. associated with symptoms and/or signs of brainstem, cerebellar and/or cervical cord dysfunction

B. Cerebellar tonsillar herniation as defined by one of the following on craniocervical MRI:

1. $\geq 5 \mathrm{~mm}$ caudal descent of the cerebellar tonsils

2. $\geq 3 \mathrm{~mm}$ caudal descent of the cerebellar tonsils plus at least one of the following indicators of crowding of the subarachnoid space in the area of the craniocervical junction:

a) compression of the CSF spaces posterior and lateral to the cerebellum

b) reduced height of the supraocciput

c) increased slope of the tentorium

d) kinking of the medulla oblongata

C. Evidence of posterior fossa dysfunction, based on at least two of the following:

1. otoneurological symptoms and/or signs (eg, dizziness, dysequilibrium, sensations of alteration in ear pressure, hypacusia or hyperacusia, vertigo, down-beat nystagmus, oscillopsia)

2. transient visual symptoms (spark photopsias, visual blurring, diplopia or transient visual field deficits)

3. demonstration of clinical signs relevant to cervical cord, brainstem or lower cranial nerves or of ataxia or dysmetria

D. Headache resolves within 3 months after successful treatment of the Chiari malformation

Figure 10: Headache attributed to Chiari malformation type I (CM1) $[7.7]^{29}$

phatic hydrops or intracranial hypertension-like). ${ }^{55}$ Presumably, the intricate relationship between valsalva and headache correlates with increases in intracranial pressure due to obstruction of CSF outflow at the level of the foramen magnum. Headaches are often occipital or sub-occipital, may be precipitated by valsalva or exertion, and may be indistinguishable from primary valsalva-induced headaches.

Both the criteria for diagnosis of Chiari malformations (clinical and radiologic) and the appropriate treatment (indications, timing and technique) are controversial. The IHS classification for headaches attributable to Chiari 1 malformation [7.7] suggests firm adherence to the new clinical and radiologic criteria before considering surgical intervention but acknowledges that future revisions to the criteria may be warranted following further prospective studies with long-term surgical outcomes. 


\section{Conclusions}

The International Classification of Headache Disorders galvanized the headache community and stimulated nosologic, epidemiologic, pathophysiologic, and genetic research. It also facilitated multinational clinical drug trials which have formed the basis of current treatment guidelines. These unprecedented and rapid advances in knowledge led to the inclusion of a number of new and well-defined primary and secondary headache disorders in the revised classification. Neurologists should be aware of these disorders to enable accurate diagnosis and should continue to consult the IHS criteria to ensure accurate diagnoses, refine the diagnostic criteria, and to contribute to the body of knowledge necessary to make further advances in the classification.

\section{REFERENCES}

1. Rasmussen BK. Epidemiology of headache. Cephalalgia 2001;21(7):774-777.

2. Mannix LK. Epidemiology and impact of primary headache disorders. Med Clin North Am 2001;85(4):887-895.

3. Cherry DK, Curt CW, Woodwell DA. National Ambulatory Medical Care Survey: 2001 summary. Advance Data 2003;337:1-44.

4. Menken M. The ambulatory workload of office-based neurologists: implications of the national ambulatory medical survey. Arch Neur 1996;53(4):379-381.

5. Sempere AP, Mola S, Medrano V, et al. Descriptive epidemiology of ambulatory neurological care in the Vega Baja (Alicante) area. Rev Neurol 2002;35(9):822-826.

6. Rajput AH, Uitti RJ, Rajput AH. Neurological disorders and services in Saskatchewan - a report based on provincial health care records. Neuroepidemiology 1988;7:145-151.

7. Isler H. Headache classification prior to the Ad Hoc criteria. Cephalalgia 1993;13(Suppl2):9-10.

8. Gobel H. Classification of headache. Cephalalgia 2001;21:770-773.

9. Ad Hoc Committee on Classification of Headache of the National Institute of Health. Classification of headache. JAMA 1962;179:717-718.

10. Headache Classification Committee of the International Headache Society. Classification and diagnostic criteria for headache disorders, cranial neuralgias and facial pain. Cephalalgia 1988;8(Suppl 7):1-96.

11. Olesen J, Rasmussen BK. Classification of primary headaches. Biomed \& Pharmacother 1995;49:446-451.

12. Olesen J. The International Headache Society classification and diagnostic criteria are valid and extremely useful. Cephalagia 1996;16:293-295.

13. Colina-Garcia RC, Carrasquero-Arias SL. Diagnostic value of the criteria of the International Headache Society in the differential diagnosis of primary headaches. Rev Neurol 2003;36:903-906.

14. Granella F, D'Alessandro R, Manzoni GC, et al. International Headache Society classification: interobserver reliability in the diagnosis of primary headaches. Cephalagia 1994;14:16-20.

15. Leone M, Filippini G, D'Amico D, et al. Assessment of International Headache Society diagnostic criteria: a reliability study. Cephalalgia 1994;14:280-284.

16. Pearce JMS. Are the International Headache Society criteria for headache useful? Cephalagia 1996;16:289-293.

17. Silberstein SD, Lipton RB, Solomon S, et al. Classification of daily and near daily headaches: proposed revisions to the HIS classification. Headache 1994;34:1-7.

18. Manzoni GC, Granella F, Sandrini G, et al. Classification of chronic daily headache by International Headache Society criteria: limits and new proposals. Cephalalgia 1995; 15:37-43

19. Silberstein SD, Lipton RB, Sliwinski M. Classification of daily and near-daily headaches: field trial of revised HIS criteria. Neurology 1996;47(4):871-875.

20. Bigal ME, Sheftell FD, Rapoport AM, et al. Chronic daily headache in a tertiary care population: correlation between the International Headache Society diagnostic criteria and proposed revisions of criteria for chronic daily headache. Cephalalgia 2002;22:432438.

21. Olesen J, Rasmussen BK. The International Headache Society classification of chronic daily and near-daily headaches: a critique of the criticism. Cephalalgia 1996;16:407-411.

22. Winner P, Wasiewski W, Gladstein J, et al. Multicenter prospective evaluation of proposed pediatric migraine revisions to the HIS criteria. Pediatric Headache Committee of the American Association for the Study of Headache. Headache 1997;37:545548.

23. Maytal J, Young M, Shecter A, et al. Pediatric migraine and the International Headache Society criteria. Neurology 1997;48:602607.

24. Leone M, D'Amico D, Grazzi L, et al. Cervicogenic headache: a critical review of the current diagnostic criteria. Pain 1998;78:1-5.

25. Sjaastad O, Fredriksen TA, Pfaffenrath V. Cervicogenic headache: diagnostic criteria. Headache 1998;38:442-445.

26. Rains JC, Penzien DB, Lipchick GL, et al. Diagnosis of migraine: empirical analysis of a large clinical sample of atypical migraine (HIS 1.7) patients and proposed revision of HIS criteria. Cephalalgia 2001;21:584-595.

27. ICD-10 Guide for Headaches. Guide to the classification, diagnosis and assessment of headaches in accordance with the tenth revision of the International Classification of Diseases and related Health Problems and its application to neurology. Cephalalgia 1997;17(Supp 19):1-91.

28. Van Drimmenlen-Krabbe JJ, Bradley WG, Orgogozo JM, et al. The application of the International Statistical Classification of Diseases to neurology: ICD-10 NA. J Neurol Sci 1998;161:2-9.

29. Headache Classification Subcommittee of the International Headache Society. The International Classification of Headache Disorders 2nd ed. Cephalalgia 2004;24(Suppl 1):1-160.

30. Saper JR. Medicolegal issues: headache. Neurol Clin 1999;17:197214.

31. Mathew NT, Reuveni U, Perez F. Transformed or evolutive migraine. Headache 1987;27:102-106.

32. Tepper SJ. Analgesic overuse is a cause of chronic daily headache. Headache 2002;42:543-547.

33. Dodick DW. Analgesic overuse is not a cause of chronic daily headache. Headache 2002;42;547-554.

34. Capobianco DJ, Swanson JW, Dodick DW. Medication-induced (analgesic rebound) headache: historical aspects and initial descriptions of the North American experience. Headache 2001;41:500-502.

35. Bahra A, Walsh M, Menon S, et al. Does chronic daily headache arise de novo in association with regular use of analgesics? Headache 2003;43;179-190.

36. Dowson AJ, Dodick DW, Limmroth V. Medication overuse headache in patients with primary headache disorders: epidemiology, management and pathogenesis. CNS Drugs 2003; In press.

37. Matharu MS, Cohen AS, Boes CJ, Goadsby PJ. Short-lasting unilateral neuralgiform headache with conjunctival injection and tearing syndrome: a review. Curr Pain Headache Rep 2003;7:308-318.

38. Elias WJ, Burchuek KJ. Trigeminal neuralgia and other neuropathic pain syndromes of the head and face. Curr Pain Headache Rep 2002;6:115-124.

39. Newman LC. Effective management of ice pick pains, SUNCT, and episodic and chronic paroxysmal hemicrania. Curr Pain Headache Rep 2001;5:292-299.

40. Peres MF. Hemicrania continua: recent treatment strategies and diagnostic evaluation. Curr Neurol Neurosci Rep 2002;2:108-113.

41. Dodick D. Hemicrania continua: diagnostic criteria and nosologic status. Cephalalgia 2001;21(9):869-872.

42. Eross EJ, Swanson JW, Dodick DW. Hemicrania continua: an indomethacin-responsive case with an underlying malignant etiology. Headache 2002;42(6):527-529.

43. Dodick DW. Thunderclap headache. J Neurol Neurosurg Psychiatry 2002;72(1):6-11. 
44. Dodick DW. Thunderclap headache. Curr Pain Headache Rep 2002;6:226-232.

45. Day JW, Raskin NH. Thunderclap headache: symptom of unruptured cerebral aneurysm. Lancet 1986;2(8518):1247-1248.

46. Raps EC, Rogers JD, Galetta SL, et al. The clinical spectrum of unruptured intracranial aneurysms. Arch Neurol 1993;50(3):265-268.

47. Wijdicks EFM, Kerkhoff H, Van Gijn. Long term follow-up of 71 patients with thunderclap headache mimicking subarachnoid hemorrhage. Lancet 1988;2:68-70.

48. Dodick DW, Eross EJ, Parish JM. Clinical, anatomical, and physiologic relationship between sleep and headache. Headache 2003;43(3):282-292

49. Dodick DW, Mosek AC, Campbell JK. The hypnic ("alarm clock") headache syndrome. Cephalalgia 1998;18(3):152-156.
50. Pascual J, Valle N. Pseudomigraine with lymphocytic pleocytosis. Curr Pain Headache Rep 2003;7:224-228.

51. Rozen TD. New daily persistent headache. Curr Pain Head Rep 2003;7:218-223.

52. Lance JW, Lambros J. Unilateral exertional headache as a symptom of cardiac ischemia. Headache 1998;38:315-316.

53. Lipton R, Lowenkopf $\mathrm{T}$, Bajwa Z, et al. Cardiac cephalgia: a treatable form of exertional headache. Neurology 1997;49:813816.

54. Mokri B. Headaches caused by decreased intracranial pressure: diagnosis and management. Curr Opin Neur 2003;16:319-326.

55. Taylor FR, Larkins MV. Headache and Chiari 1 malformation: clinical presentation, diagnosis, and controversies in management. Curr Pain Headache Rep 2002;6:331-337. 